\section{La teoría hegeliana de la economía moderna}

The Hegelian Theory of Modern Economics

\author{
Erzsébet Rózsa
}

\section{RESUMEN}

La tesis principal de este artículo sostiene que la teoría hegeliana de la economía moderna debe entenderse como una filosofía económica, y no como una economía. Hegel no fue un economista como Smith, Say o Ricardo, sino que elaboró un tipo diferente de teoría económica que, como interpretación igualmente precisa, merece mucha más atención de la que ha recibido hasta ahora. El único intérprete que habló sobre la teoría de la economía moderna y la filosofía económica hegeliana fue Althusser, que utilizó esta expresión en un sentido más bien peyorativo. La teoría económica de Hegel fue recibida por muchos filósofos, desde Gans y Marx, a Lukács a Marcuse, aunque la falta de claridad sobre la relación entre la economía y la teoría filosófica de la economía moderna significó que Hegel fuera medido según los estándares de teorías económicas como las de Smith, Ricardo o Marxy, en consecuencia, condenado o alabado. Su logro en el campo de la teoría económica de la modernidad quedó en la oscuridad en la medida en que su teoría de la sociedad burguesa y su significado fue reemplazada por su filosofía política. El objetivo de este artículo es mostrar las características específicas de la filosofía económica de Hegel.

Palabras clave: Hegel; Modernidad; Economía; Filosofía económica; Sociedad burguesa

\section{ABSTRACT}

The main thesis of this article maintains that the Hegelian theory of modern economics should be understood as an economic philosophy, and not as an economy. Hegel was not an economist like Smith, Say, or Ricardo, but he produced a different kind of economic theory which, as an equally accurate interpretation, deserves much more attention than it has received thus far. The only interpreter who spoke about modern economic theory and Hegelian economic philosophy was Althusser, who used this expression in a rather pejorative sense. Hegel's economic theory was received by many philosophers, from Gans and Marx, to Lukács to Marcuse, but the lack of clarity about the relationship between economics and the philosophical theory of modern economics meant that Hegel was measured by the standards of economic theories such as those of Smith, Ricardo or Marx, and consequently condemned or praised. His achievement in the field of the economic theory of modernity was left in the dark as his theory of bourgeois society and its meaning was superseded by his political philosophy. The aim of this article is to show the specific characteristics of Hegel's economic philosophy.

Keywords: Hegel; Modernity; Economy; Economic Philosophy; Civil Society
INFORMACIÓN

http://doi.org/10.46652/resistances.v2i4.64

ISSN 2737-6222 |

Vol. 2 No. 4, 2021, e21064

Quito, Ecuador

Enviado: octubre 18, 2021

Aceptado: diciembre 05, 2021

Publicado: diciembre 13, 2021

Publicación continua

Sección dossier | Peer Reviewed

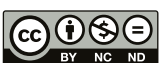

open@access

AUTOR

Erzsébet Rózsa

Universidad de Debrecen - Hungría rozsa.erzsebet@arts.unideb.hu

Conflicto de intereses

El autor declara que no existe conflicto de interés posible.

Financiamiento

No existió asistencia financiera de partes externas al presente artículo.

Agradecimiento

N/A

Nota

Este artículo ha sido traducido desde el alemán por Angelo Narváez León (UCSH).
PUBLISHER 


\section{Hegel: ¿̇economista o filósofo de la economía?}

Cuando Birger Priddat publicó en 1990 el libro Hegel als Ökonom, le criticó a Charles Taylor haber "revestido a Steuart y Smith con el ropaje del período clásico inglés. Ambos son británicos, pero sus concepciones económicas son contrarias. La tensión entre la disposición económicocameralista estatal de la economía alemana del siglo XVIII, que aparece en la economía política de Steuart, y la joven economía clásica inglesa renovada, o ciencia económica de principios del siglo XIX, determina la recepción hegeliana de la economía mucho más allá de una mera preocupación por los clásicos. Hegel encontró en Steuart un analista de los procesos de mercado que se destacó en la economía del siglo XVIII, aunque su posición económica inicial en principio no se distinguiera de Justi o Sonnenfels, por nombrar a los autores cameralistas más conocidos de la época” (1990, p. 10). Priddat continúa diciendo que, “en la práctica, Hegel trata la 'economía política' -el sistema de necesidades- como un sistema propio de la teoría económica pura, mientras que ‘la policía y la corporación’ pertenecen a las ciencias económicas” (1990, p. 14); y luego, afirma que "de acuerdo al modo y la manera de su articulación de los asuntos económicos, Hegel pertenece evidentemente al espectro más antiguo de la economía alemana -al campo transitorio del cameralismo a la economía política- antes de que se codificara como ciencia económica” (1990, p. 15, el destacado es nuestro). Por lo tanto, concluye Priddat, "la lectura de Hegel de la economía clásica sigue siendo cameralista" (1990, p. 18).

Esto es correcto en la medida que la recepción hegeliana de la economía política es mucho más que una mera preocupación por “los clásicos ingleses”, aunque no solo en el sentido enfatizado por Priddat. Hegel elaboró su teoría no sólo con respecto a la "disposición cameralista de la economía alemana”, sino principalmente en relación a la economía británica (“clásica inglesa”, a decir de Priddat). Desde la perspectiva de la historia de las ideas, tiene sentido preguntarse hasta qué punto la formulación de Hegel está vinculada con ciertos aspectos de la economía cameralista alemana o de la economía británica. Sin embargo, en última instancia, la teoría de Hegel no puede clasificarse ni en el "espectro más antiguo de la economía alemana" ni en el período “clásico inglés”. Es por esta razón que el foco temático de este artículo está en otro lugar: a saber, en el momento donde sale a la luz la interpretación de Hegel como una teoría autónoma de la economía moderna.

Como aclaración de esta perspectiva analítica, remito a mi trabajo de 1984 escrito en húngaro, titulado La filosofía económica de Hegel (1993). En mi opinión, la idea central de esa monografía sigue siendo válida en la actualidad: la teoría hegeliana de la economía moderna no debe describirse principalmente como una economía -sea cameralista, política, nacional, estatal o científica- sino como una teoría filosófica de la economía de la modernidad. Desde esta perspectiva analítica es evidente que el problema con la exposición de Priddat estriba en que la concepción de Hegel queda unilateralmente situada en la tradición cameralista alemana de la economía. Otro problema radica en una demarcación estricta de la "economía clásica inglesa", que no estriba simplemente en un asunto de puntos de vista históricos, o de su consistencia en la historia de las ideas, sino en los diferentes enfoques teórico-metodológicos de prácticamente los mismos temas.

Es indiscutible que Hegel tuviera la intención de abordar la economía como una ciencia particular, ya que trabajó de manera intensiva los asuntos económicas ya desde la época de Berna hasta 
los últimos años en Berlín; sin embargo Hegel no fue un economista como Say o Ricardo, a los que menciona en la Filosofía del derecho, o como Steuart, sobre cuya obra escribió un comentario perdido; sino que, en realidad, elaboró una teoría económica distinta, e incluso diferente, que no es disciplinalmente científica sino filosófica.

Antes de exponer los detalles de esta teoría, resulta provechoso echar un vistazo a algunos de los puntos centrales de la historia de la recepción de la interpretación hegeliana de la economía. En ese horizonte, fue Louis Althusser quien utilizó la expresión "filosofía económica" en un sentido peyorativo para referirse a la concepción hegeliana. La filosofía económica de Hegel, según el reproche de Althusser, se basa en una "antropología ingenua" (Althusser, 1968). Sin embargo, la explicación simplista y despectiva de Althusser no es de ninguna ayuda. La visión de Hegel de la economía moderna fue retomada en los siglos XIX y XX por filósofos profundamente interesados en la economía, entre ellos por el estudiante de Hegel, Eduard Gans; el estudiante de Gans, Karl Marx, y más tarde, por Georg Lukács y Herbert Marcuse. En Lukács la teoría de Marx adquirió un estatuto prioritario para explicar la concepción de Hegel de la economía y la sociedad modernas. Pero en realidad, a Lukács no le interesaba la influencia teórica de la economía en la filosofía práctica de Hegel, sino sobre todo la "fecundidad" de esa "ciencia fundamental de la vida social” para la dialéctica marxista (Lukács, 1948, p. 23-26). La cuestión del vínculo Marx-Hegel en relación con las categorías económicas debería plantearse de manera fundamentalmente diferente a como lo hizo Lukács. En la economía de Marx, la teoría de la plusvalía se entiende como la novedad más importante, cuyo secreto había sido revelado en su obra principal El capital, específicamente en el apartado sobre la compra y venta de trabajo. Ahí la fuente de la plusvalía no es el trabajo mismo, sino la fuerza de trabajo o la capacidad de trabajar. Sin embargo, la distinción entre trabajo y fuerza de trabajo, es decir una de las novedades decisivas de la economía marxista, se remonta a los análisis de Hegel especialmente en el §61 de la Filosofía del derecho sobre el uso de la cosa, en el §67 sobre la enajenación de la propiedad, y a su análisis de la fuerza y de las formas fenoménicas en la Lógica. Marx solo se refirió a la Filosofía del derecho de Hegel en una breve nota a pie de página, pero sin duda se benefició enormemente de su lectura. En los filósofos antes mencionados, así como en las interpretaciones marxistas de la segunda mitad del siglo XX, la distinción de Hegel entre la economía como ciencia particular y una posición filosófico-económica se perdió por completo. Esto es tanto más sorprendente, si no del todo incomprensible, dado que la filosofía marxista tenía fuertes pretensiones socio-filosóficas. La teoría de Hegel tematiza otra dirección en la historia de la interpretación en el marco de su filosofía política, e incluso la disuelve en ella, como bien muestra Rolf-Peter Horstmann en "Hegels Theorie der bürgerlichen Gesellschaft". Horstmann toma como idea clave de la sociedad burguesa la siguiente pregunta: “¿en qué se basa el significado de la teoría de la sociedad burguesa para la filosofía del Estado de Hegel?”. Para él, el factor decisivo estriba en cómo Hegel integra la sociedad burguesa en su filosofía política, y qué función política le atribuye (Horstmann, 2014, p. 194). Ante esta consideración se puede decir por ahora lo siguiente: en la historia de la recepción y el impacto de la Filosofía del derecho de 1820, difícilmente se pueda reconocer del todo el logro filosófico singular de Hegel en su interpretación de la economía moderna. Por ejemplo, Klaus Vieweg se refirió a elementos de la "economía” de Hegel en su extensa monografía, aunque no vio ni reconoció la innovación filosófica propia de 
Hegel y sus logros en la interpretación de la economía moderna, de gran importancia para su teoría de la modernidad (Vieweg, 2019, pp. 133-508).

Por otro lado, Manfred Riedel mostró un trasfondo más amplio en la historia de las ideas cuando incluyó la concepción aristotélica del oikos y las teorías modernas del derecho natural en su relectura de la teoría hegeliana de la sociedad burguesa como los puntos centrales de su interpretación de la economía moderna (Riedel, 1969, pp. 76-99; 1975, pp. 247-275). En los Studien zu Hegels Rechtsphilosophie, Riedel analiza "la disciplina de la filosofía práctica fundada por Aristóteles" y "su tradición en la Edad Media y los albores de la modernidad”. Ahí Riedel enfatiza los logros de Hegel en la renovación de la filosofía práctica más allá de Kant. El "método dialéctico" de Hegel va más allá de la tradición escolástica de la filosofía práctica, partiendo del concepto kantiano de libertad y del descubrimiento de la espontaneidad de la conciencia del yo en Kant. Las condiciones que hicieron esto posible para Hegel fueron:

i) La idea kantiana de la autonomía de la voluntad, a la que Hegel adhiere,

ii) La inclusión de la economía en la construcción de la filosofía práctica, además del intento de incorporar sus principios en la experiencia históricamente contemporánea del mundo social e industrial; y,

iii) Su conexión con la dimensión de la historia (Riedel, 1969, pp. 14-17).

El mérito de Riedel consiste principalmente en haber visto en la teoría hegeliana de la sociedad burguesa una dimensión de su "filosofía práctica", cuya rehabilitación en los años ochenta se produjo precisamente gracias a Riedel (1972, ff). Ahora, el esfuerzo de Riedel también puede tener un efecto inspirador en la filosofía práctica contemporánea (Siep, 2010; Weisser-Lohmann, 2011). Para rehabilitar la concepción hegeliana de la sociedad burguesa en los Studien zu Hegels Rechsphilosophie, una de las finalidades de Riedel, no era en estricto rigor necesario examinar en detalle la teoría de la economía moderna de Hegel en su diferencia con la economía británica como ciencia particular o demostrar su originalidad filosófica (Riedel, 1969, p. 135). Sin embargo, su observación se refiere a esta línea de investigación e interpretación cuando enfatiza que a Hegel le preocupaba la “inclusión de la economía en la construcción de la filosofía práctica”, es decir, en "el intento de incorporar sus principios en la experiencia históricamente contemporánea del mundo social e industrial” Riedel, 1969, p. 17). Es precisamente en el marco de la construcción de la filosofía práctica hegeliana donde resulta posible diferenciar entre la economía como una ciencia particular con una tradición propia, y una teoría de la economía moderna como reflexión filosófica sobre la experiencia contemporánea del mundo social e industrial vinculada con una nueva relación histórica. Eso sí, la lectura de Riedel no tuvo un efecto notable en la recepción de la obra de Hegel.

La continua falta de claridad sobre la relación entre la economía y la teoría filosófica de la economía moderna produjo que se midiera a Hegel con el criterio de la economía y, en consecuencia, se lo condenara o elogiara. Cabe precisar aquí casi toda la tradición marxista consideró que Hegel tomó el punto de vista de los economistas que no asumían como punto de partida el trabajo y la producción sino las necesidades y, por tanto, el consumo (en palabras de Hegel: el consumir). 
Por su parte, Priddat ha reinterpretado el juicio de Marx según el cual Hegel se sitúa en el punto de vista de la economía moderna o de la economía política de su tiempo:

...cuando sintetiza la economía smithiana de mercado (el sistema de las necesidades) con la policía y corporación cameralista, Hegel se encuentra -para que se entienda la tesis corregida- en el plano de la economía de su tiempo, aunque no al nivel inglés, sino alemán (Priddat, 1990, p. 11).

Por supuesto que, si se intenta interpretar la sociedad burguesa desde esta posición económica, se puede aceptar a primera vista esta afirmación en la medida que se plantea efectivamente la problemática de las necesidades de los trabajadores.

Ahora bien, de una inspección más cercana resulta que la teoría hegeliana de las necesidades también tiene una configuración estructural diferente, lo que ofrece una posibilidad de interpretación distinta y aún más plausible para el problema en cuestión. En los §§182-188 de la Filosofía del derecho, es decir en el contexto de la sociedad burguesa, el análisis del sistema de la necesidades está precedido de consideraciones importantes que van más allá del problema económico de las necesidades y el consumo, pero también del trabajo y de la producción. En estos parágrafos introductorios al capítulo sobre la sociedad burguesa, especialmente en los $\$ 185$ y §187, Hegel aborda ambos principios (Hegel, 1986, VII, §182-86), así como las estructuras básicas o movimientos fundamentales de la economía y la sociedad modernas correspondientes a estos principios. La sociedad burguesa, que constituye el concepto integral de la economía y la sociedad modernas, es caracterizada en el \$185 como “el espectáculo del libertinaje y la miseria, con la corrupción física y ética que es común a todos” (Hegel, 1986, VII, §185). En el $\$ 187$ Hegel introduce la cultura en relación al trabajo, y combina estos enfoques con el principio sistemático históricamente conformado de los "tiempos modernos" o la "libertad subjetiva" de todos (Hegel, 1986, VII, §185). No es difícil ver que estas consideraciones sistemáticas, que preceden al sistema de necesidades, van mucho más allá del problema de las necesidades, que es otra razón por la que no pueden explicarse únicamente con los instrumentos de la economía como ciencia especializada, sino que se necesita otro tipo de herramienta teórica para explicarlo adecuadamente. La posición esbozada por Hegel en los párrafos introductorios de la sociedad burguesa, así como su lenguaje y el uso de expresiones como "espectáculo”, “corrupción” o "estado del entendimiento", evidencian que no deben entenderse como categorías económicas, porque su significado en términos de contenido sólo puede explicarse en un horizonte de interpretación diferente y con un conjunto distinto de instrumentos conceptuales.

Para mostrar en términos concretos la diferencia teórico-metodológica fundamental entre la economía y la filosofía económica en Hegel, primero nos ocuparemos del derecho abstracto, el primer capítulo de la Filosofía del derecho. Este es un ejemplo de cómo la concepción hegeliana del derecho abstracto no solo se relaciona con las teorías del derecho natural de Rousseau, Montesquieu y Kant, sino también con temas y categorías económicas. La posesión y la propiedad como categorías jurídicas también adquieren aquí un contenido económico que, sin embargo, no puede explicarse en términos puramente económicos. Incluso antes el joven Hegel ya se 
había preocupado por conceptos económicos y legales como la posesión y la propiedad. En la Filosofía del espíritu de Jena vinculó estos conceptos económicos con el amor como primera forma de intersubjetividad (Rózsa, 2014). Entonces, ¿de qué se trata todo esto realmente?

\subsection{El derecho abstracto desde la perspectiva de la filosofía económica.}

En el apartado sobre el derecho abstracto, Hegel puso en primer plano la relación entre el yo como personalidad o persona jurídica, y la cosa. En el lenguaje de la economía marxista, esta relación corresponde a los prerrequisitos de la producción simple de mercancías, aunque dentro de este marco las expresiones que utilizó Hegel, tales como posesión, propiedad, apropiación corporal, elaboración, uso de la cosa, alienación, o la distinción entre el valor de uso y el valor de la cosa más allá del sentido que tienen en su dimensión jurídica, también reciben significados que no pueden ser simplemente calificados como económicos. El posicionamiento hegeliano sobre la relación entre derecho y economía pone de manifiesto la preeminencia de la persona jurídica sobre el burgués, y también de las disposiciones legales sobre el orden económico. Esta relación sólo se explica más adelante en el capítulo sobre la sociedad burguesa "verdadera", y se revela como la apariencia invertida de las estructuras "verdaderas" del mundo moderno. Precisamente en el trasfondo de esta estructura no se reconoce simplemente una posición solo económica o jurídica positiva, sino la propia posición filosófica de Hegel sobre el problema general de la economía y la sociedad modernas. En consecuencia, el tema del derecho abstracto aparece bajo una luz diferente desde la perspectiva (desde el "punto de vista") de la sociedad burguesa: el derecho (abstracto) -más allá de sus cuestiones jurídicas y filosófico-jurídicas - también proporciona la (primera) forma de determinación de las condiciones económicas de los "nuevos tiempos" en relación al "mundo moderno" diferenciado (legal, social, política, privada y públicamente). En este sentido, el derecho se muestra como la determinación formal de las relaciones económicas y sociales que, sin embargo, como resultado de la investigación de las complejas relaciones entre las formas diferenciadas de la sociedad y la economía modernas, sólo puede ser explicado más adelante en el capítulo sobre la sociedad burguesa (Hackenesch, 2000, p. 95).

Por supuesto, esto requiere una observación sobre la relacionalidad como base metodológica de Hegel. Hackenesch discute la relacionalidad en el contexto de la Lógica, cuya caracterización fundamental son las relaciones entre los términos que deben entenderse como mediación en cuanto "relacionalidad absoluta". El sentido debe constituirse precisamente en esta mediación con respecto a la relacionalidad absoluta, por eso según Hackenesch (2000, p. 108) el significado no puede separarse del sistema. Robert Brandon entiende la relacionalidad de Hegel de manera diferente, porque la ve como una variante del expresivismo con el que Hegel aborda el conceptualismo no solo pragmática e inferencialmente, sino también relacionalmente en el sentido de que constituye lo implícito y lo explícito a través de su mutua relación expresiva (Brandon, 2001, p. 53). Cabe precisar que, si se diferencian las relaciones externas e internas en Hegel, y se puede hacer con justa razón, entonces se produce una estructura dinámica de relacionalidad (o metamorfosis) que también caracteriza cambios de significado que afectan el impacto de la expresividad comunicativo-lingüística. Una relación externa, como con la naturaleza exterior, se internaliza y viceversa: por ejemplo, el propósito inmediato de satisfacer 
mis necesidades se convierte en una relación inicialmente externa con los demás. La expresividad de estas relaciones es particularmente importante para el joven Hegel, por ejemplo, en la Filosofía del espíritu de Jena (Rózsa, 2014).

Volviendo sobre el problema anterior; detrás del punto de partida de la persona jurídica, por un lado, y de la forma jurídica de la relación económica por otro, hay en el derecho abstracto un prerrequisito antropológico que Hegel resume así: "como persona tengo mi vida y cuerpo al mismo tiempo, como otras cosas, sólo en la medida en que sea mi voluntad" (Hegel, 1986, VII, §47). La vida y el cuerpo, en tanto naturales, y el espíritu en cuanto determinación sociocultural, expresan a la vez los aspectos físico-biológico-antropológicos y los aspectos antropológicos socialmente contextualizados del ser humano (Hegel, 1986, VII, §48). Como ha mostrado Dean Moyar (2002), la complementariedad como figura metodológica del pensamiento de Hegel también puede reconocerse en esta estructura del alma y del cuerpo en relación con la voluntad como espíritu. Ahora bien, la complementariedad se reinterpreta o amplía en el presente estudio, porque como he dicho en otra parte (Rózsa, 2007, p. 12), expresa también un elemento estructural fundamental de la relacionalidad hegeliana en el ámbito de lo práctico en general. Es precisamente en este contexto específico donde Hegel situó esta relación antropológica entre lo natural, lo espiritual y cultural como la base teórico-conceptual para explicar la relación entre el hombre y las cosas (particulares y en general), y también para explicar el contenido y las relaciones económicas. Por supuesto, la interpretación pragmática de la relación entre naturaleza y espíritu como cultura en Hegel, tal como la entiende Brandon, puede ser inspiradora para la reconstrucción del "Hegel auténtico", a decir de Gadamer (Brandon, 2001, p. 54 y ss.). Sin embargo, el "expresivismo pragmático" de Brandon debería complementarse, en cualquier caso, por ejemplo, con el "expresivismo racionalista" de Hegel para entender la relación entre la naturaleza y el espíritu como cultura, según el mismo Brandon enfatiza. Esto es así porque Hegel destaca que soy libre para el otro en mi cuerpo, que es también mi voluntad (Hegel, 1986, VII, §48). En esta libertad en el cuerpo como voluntad reside también "la relación anticipada con los demás", como una de las primeras formas de la intersubjetividad en la existencia humana (Hegel, 1986, VII, §51). Esta intersubjetividad como aspecto y componente del Dasein en cuanto “existencia” del individuo se institucionaliza como reconocimiento en el contrato, y se implementa en actividades económicas de enajenación e intercambio legalmente aseguradas. En el trasfondo de estas relaciones y actividades/prácticas legalmente reguladas y legitimadas, y al mismo tiempo económicamente determinadas que se problematizan en el marco del derecho abstracto en relación con la persona, es decir en última instancia la libertad infinitamente subjetiva de todos, también puede reconocerse como el principio histórico más elevado del mundo moderno (Hegel, 1986, VII, §51). Esta compleja ubicación (jurídica, económica, antropológica, histórica) de las categorías de la economía en el derecho abstracto remite a un horizonte de interpretación multidimensional (Tillich, 1962; Rózsa, 2005, pp. 72) que presupone estructuras relacionales ab ovo (como el cuerpo y la voluntad, o el reconocimiento). La posición esbozada por Hegel expresa una posición teórica integral que va más allá de la economía (así como de las ciencias jurídicas). De esta manera ya se puede ver en el derecho abstracto que Hegel reinterpreta las categorías económicas desde el principio y, en consecuencia, hablamos en el primer capítulo de una teoría de la economía filosóficamente configurada. 


\subsection{Consecuencias metodológico-teoréticas.}

Hegel no solo utilizó categorías económicas en el derecho abstracto, sino también en la moralidad y la eticidad donde adquieren significados ulteriores. Esto no puede explicarse en detalle hic et nunc, aunque por ahora es importante una consecuencia: la posición relacional multidimensional de interpretación antes mencionada permite e incluso requiere que la concepción hegeliana no sea entendida como una economía, sino como una teoría filosófica que podemos llamar filosofía económica (Rózsa, 2012). En esa compleja perspectiva esbozada que impregna la estructura general y el tema de la Filosofía del derecho como obra principal de su teoría de la economía y de la sociedad moderna, resulta sumamente problemático comparar su formulación de manera inmediata e indiferenciada con la opinión de los economistas, o incluso clasificar una u otra posición por encima o por debajo de otra. Esto se debe precisamente a que Hegel se ocupa de diferencias sustantivas y metodológicas entre la economía como ciencia particular o ciencia especializada (como la psicología, la historia, el derecho, etc.), y la filosofía económica o las ciencias filosóficas en general. Esta precisión representa una gran diferencia entre Kant y Hegel. Kant utilizó las ciencias particulares como la física y las matemáticas en tanto modelos para su filosofía teórica crítica, pero no encontró un modelo análogo en el ámbito de la filosofía práctica; así, por ejemplo, una referencia a la Biblia no puede asumir esa función. Esta diferencia entre Kant y Hegel puede tener algo que ver con el hecho de que las ciencias (del espíritu) particulares comenzaron a independizarse en la época de Hegel. Este desarrollo puede ser bien ejemplificado por la distinción hegeliana entre las formas de narración histórica en las lecciones sobre filosofía de la historia universal.

De lo dicho hasta ahora se desprende que para la reconstrucción de la interpretación hegeliana de la economía moderna, también deben responderse preguntas que van más allá de los temas técnicos de la economía. Algunas de estas “meta-preguntas” son las siguientes: ¿por qué desarrolló Hegel su propia teoría de la economía?, ¿fue debido a restricciones del sistema o por razones conceptuales o relacionadas con el contenido?, ¿puede la filosofía económica decir algo sobre temas en muchos aspectos comunes, pero que la economía no logra referir?, ¿debe entenderse su filosofía económica como una teoría relevante de la economía moderna?, y si es así, ¿cómo se relaciona con la economía?

Por supuesto que no se puede recapitular todo el concepto en un artículo. Es por eso que aquí nos limitaremos a algunos puntos específicos. Primero ofreceremos una descripción general de la teoría sistemática de la economía moderna de Hegel. Segundo, discutiremos la educación como un concepto de la filosofía económica, en el que se puede reconocer uno de los logros específicos de la teoría de Hegel, y en ese vínculo quedará claro que Hegel fue de hecho un filósofo económico, en la medida en que fue el único filósofo de su tiempo que vinculó estrechamente la economía británica con la concepción alemana de la burguesía educada y elaboró una concepción autónoma de la economía moderna. 


\section{La filosofía económica hegeliana como teoría soberana de la economía y la sociedad moderna.}

\subsection{La filosofía económica sistemática: una teoría relevante de la economía moderna.}

Hegel explicitó esta concepción en todas las partes de la Filosofía del derecho. Esto quiere decir que cada capítulo de la obra debe interpretarse como un componente de su filosofía económica, y solo de esta manera parece comprensible que Hegel piense, por ejemplo, los elementos de la producción simples de mercancías en el derecho abstracto, el bienestar como categoría económica en la moralidad, la nueva función económica de la familia en la vida privada y las colisiones de la economía y la sociedad modernas (pobreza y riqueza) en el Estado; y que además examine las posibilidades y los límites de la intervención estatal en los movimientos económicos y sociales (enfrentando el empobrecimiento y los extremos sociales en general). Sin embargo, en el foco temático de su concepción de la economía moderna ésta configura a la sociedad burguesa, que además se conecta históricamente con los "nuevos tiempos", los “tiempos modernos" o el “mundo moderno" (Hegel, 1986, VII, §186). En ese contexto complejo, su teoría de la sociedad burguesa es de suma importancia para su concepción general de la modernidad. Habermas ofrece una interpretación provechosa de la teoría hegeliana de la modernidad cuando dice que, "Hegel fue el primer filósofo en desarrollar un concepto claro de la modernidad", porque muestra los “requisitos previos de la autoimagen moderna” (Habermas, 1986, p. 13).

La filosofía económica también forma una de las disciplinas filosóficas de la Filosofía del derecho, que, junto con otras disciplinas filosóficas como la filosofía moral, la filosofía jurídica y política, la filosofía social y la filosofía de la historia, configuran su filosofía práctica en general. Esta composición de la filosofía práctica en la Filosofía del derecho de Hegel abre un horizonte de interpretación para la tematización de la economía moderna que la economía como ciencia particular no puede ofrecer, en la medida que no la necesita. El horizonte multidimensional de interpretación de Hegel no es relevante en realidad para las ciencias particulares como la economía, porque la economía no puede/no quiere nada con los problemas que Hegel describe, como la mala infinitud de las necesidades o el mundo fenoménico de la sociedad burguesa. Sin embargo, a continuación, explicaremos que la posición analítica compleja-sistemática de Hegel ofrece un potencial explicativo diferente pero igualmente relevante para los fenómenos, estructuras y movimientos de la economía y la sociedad modernas, que no pueden desarrollarse con los instrumentos de la economía.

Por el contrario, la filosofía económica de Hegel es una interpretación filosófica-sistemática de los fenómenos, mecanismos y estructuras de la economía moderna que se integran en la sociedad moderna. Esta interpretación, con su propio conjunto de instrumentos, representa una teoría alternativa de la economía moderna a la que ofrece la economía política británica, que no es solo diferente sino también relevante. La consideración de Hegel en el \$189 es decisiva para explicar esta separación de interpretaciones científico-económicas y filosóficas.

\subsection{La justificación hegeliana de la teoría filosófica de la economía.}

En primer lugar, conviene recordar que Hegel nunca utilizó la expresión “filosofía económica”, aunque aun así es legítimo utilizarla. Se podría argumentar que Hegel tampoco utilizó el término 
"filosofía política", pero no hay duda de que es legítimo utilizarla también. En vez de enfocarse en argumentos tan banales, se puede llamar la atención sobre un pasaje de la Filosofía del derecho para entender la filosofía económica como una expresión legítima en relación a la economía como ciencia especializada; es decir al pasaje donde Hegel tematiza la economía y la distingue de su propia interpretación de la economía moderna. En la nota al \$189, se lee lo siguiente:

\begin{abstract}
...la economía política es la ciencia que tiene en estos puntos de vista su comienzo, pero que tiene que presentar luego la relación y el movimiento de la masa de datos contingentes en su entrelazamiento o determinación cualitativa y cuantitativa. Es una de las ciencias que ha encontrado en la época moderna su terreno propio. Su desarrollo muestra el interesante proceso de cómo el pensamiento descubre, a partir de la infinita cantidad de individualidades que en un primer momento tiene ante sí, los principios simples de la cosa, el entendimiento que actúa sobre ella y la gobierna. Si bien reconocer esta apariencia de racionalidad que reside en la cosa y actúa en ella es en esta esfera de las necesidades lo que produce la conciliación, por otra parte, éste es el terreno en el que el entendimiento de los fines subjetivos y las opiniones morales descarga su descontento y su fastidio moral (Hegel, 1986, VII, §189).
\end{abstract}

Como puede verse en el texto, Hegel define la "economía política" como una ciencia que históricamente representa la primera ciencia de la economía moderna. Es "una de las ciencias que ha encontrado en la época moderna su terreno propio" (Hegel, 1986, VII, §189), y que además se relaciona con el hecho de que "la libertad de propiedad" como fundamento de la economía moderna sea también un proceso reciente (Hegel, 1986, VII, §62). Sistemáticamente, la economía es la ciencia que tiene su punto de partida inmediato en las múltiples necesidades, pero además Hegel combina esta ubicación del objeto de la economía con la interpretación filosófica de la economía moderna cuando introduce los dos principios de la sociedad burguesa en el §182. Un principio es la particularidad de las necesidades, y el otro es la universalidad de la satisfacción de las necesidades (Pippin, 2001; 2002; Pinkard, 2009; Brandon, 2002). Las expresiones "particularidad" y "universalidad" ya indican en sí mismas que se trata de una aproximación filosófica por una terminología lógico-filosófica. Porque no son los múltiples fenómenos de las necesidades y su satisfacción como una "infinita cantidad de individualidades" -que serían en sí mismas objetos económicos- los temas propiamente hegelianos aquí, sino más bien la peculiaridad adicional de estos fenómenos, que se caracterizan por los conceptos de particularidad y universalidad de las diversas necesidades y de su satisfacción. Resulta que la relación entre las categorías de la economía y los múltiples fenómenos requiere desde el principio una explicación adicional que los conceptos y principios de la filosofía pueden proporcionar. En este contexto, la "conceptualidad” (Rózsa, 2012; Hösle, 2005) de Hegel debe entenderse como una teoría filosófica lógicamente articulada y sistemáticamente explícita que se lleva a cabo en el contexto práctico en su principal obra (Vieweg, 2012; Quante, 2014), la Filosofía del derecho de 1820.

2.3. Los dos principios de la sociedad burguesa vistos desde los "principios simples de la cosa"

Un problema posterior se encuentra en el pasaje del texto donde Hegel, al caracterizar la economía, no habla de los dos principios en cuestión, sino más bien de los principios simples de la 
cosa. Tiene sentido entonces preguntarse cómo los dos principios de la sociedad burguesa, la particularidad de las necesidades y la universalidad de su satisfacción en el §182 y los "principios simples de la cosa” en el \$189, se relacionan entre sí. Además, conviene llamar la atención sobre la siguiente observación de Hegel: la economía "es la ciencia que tiene en estos puntos de vista su comienzo" (Hegel, 1986, VII, §189). ¿Cuáles son los puntos de vista de los que estamos hablando aquí, en el primer párrafo del sistema de necesidades?

En los párrafos introductorios de la sociedad burguesa que preceden al sistema de necesidades, Hegel no solo problematiza ambos principios sino también algunas características estructurales básicas de la sociedad burguesa ("estado del entendimiento", “eticidad perdida” “el espectáculo de la ruina física y moral”, la identidad ética faltante, la separación de la vida privada y pública, la educación como liberación y trabajo de liberación superior). Hegel desarrolla sus reflexiones sobre la economía solo después de problematizar estas características de la sociedad y la economía modernas. ¿Qué se supone que significa esta secuencia de ambos principios y los principios simples para la relación entre la filosofía económica y la economía?

En el cuerpo principal del §182, Hegel analiza los dos principios de la sociedad burguesa. Los términos "particularidad" y "universalidad" aquí se refieren a las necesidades y su satisfacción, que inicialmente son categorías económicas, aunque están sujetas a reinterpretaciones como “cambios de significado" debido a su inclusión en estos términos lógico-filosóficos. Por otro lado, hay algunas precisiones en la nota del §189, que constituye el texto clave para la interpretación de la economía. Ahí no se trata de los dos principios del §182, sino de “los principios simples de la cosa, el entendimiento que actúa sobre ella y la gobierna” (Hegel, 1986, VII, §182). ¿Cuál es la diferencia entonces, si tanto la filosofía como la economía abordan las necesidades y su satisfacción? La respuesta está ligada a la distinción metodológica fundamental de Hegel entre filosofía y ciencia particular, y en cómo atribuye esta distinción a la diferencia entre entendimiento y razón, que expresan dos posiciones metodológico-teóricas muy diferentes ("puntos de vista”, a decir de Hegel) sobre el área temática en cuestión: los fenómenos y mecanismos de la economía.

Hegel caracteriza la economía como una ciencia particular desde el punto de vista del entendimiento. En el horizonte de interpretación del entendimiento, la economía tematiza "la esfera de las necesidades", para "presentar luego la relación y el movimiento de la masa de datos contingentes en su entrelazamiento o determinación cualitativa y cuantitativa". Al mismo tiempo ese movimiento se describe desde una perspectiva filosófica, que representa la razón. Desde esta perspectiva filosófica, la "esfera de las necesidades" resulta ser la "apariencia de racionalidad". Por otro lado, la economía como ciencia especializada no puede reconocer esta apariencia de racionalidad en la esfera de las necesidades: tematiza las apariencias de la economía moderna como una "esfera" de fenómenos diversos incluso en su "entrelazamiento" (es decir, en sus conflictos tales como pobreza y riqueza, miseria y lujo). Pero la economía no puede entender esto como una "apariencia de la racionalidad" que está “en la cosa”, es decir en su esencia de manera activa, y precisamente por esa razón permanece inaccesible para ella. En consecuencia, en la economía como ciencia especializada no se trata del 'sistema de necesidades'. El entendimiento y sus instrumentos, las categorías, no sirven para tematizar la multiplicidad de necesidades como esfera fenoménica o sus intrincadas apariencias como estructura que 
reposa en la materia y opera según sus relaciones esenciales, y en este sentido tampoco puede interpretarlas como un sistema. El entendimiento como posición metodológica de la economía en tanto ciencia particular no revela las necesidades en sus relaciones "verdaderas" o "racionales", por lo que estamos hablando de otra cosa, de la "esfera" esencialmente aún no estructurada de necesidades diversas y sus entrelazamientos. Las estructuras y movimientos "verdaderos" de las necesidades, por otra parte, deben reconocerse y mostrarse desde el punto de vista de la razón como una posición compleja, relacional y sistemática. Precisamente por eso, desde el punto de vista de la economía, no se trata del "sistema" sino de la "esfera" de las múltiples necesidades como un mundo fenoménico, que en su propia perspectiva no debe entenderse como un sistema ni como la apariencia de lo razonable de la cosa. Por tanto, es comprensible que, en el horizonte de interpretación del entendimiento como base metodológica de la ciencia particular, la economía se muestre como "la ciencia que tiene en estos puntos de vista su comienzo", justamente porque los "puntos de vista” corresponden al procedimiento analítico del entendimiento.

La razón en cambio representa el horizonte de interpretación de la filosofía, donde los fenómenos de la economía moderna como una "infinita cantidad de individualidades" y los “principios simples" de las relaciones y movimientos de los fenómenos no se analizan del mismo modo que en la economía. Como bien muestra la sentencia de Hegel en el §189, en el horizonte filosófico de interpretación se refleja en primer lugar la posición de la ciencia que tematiza estos fenómenos y sus simples principios, es decir, la economía. Los temas de la economía, sobre todo la esfera de las necesidades, se muestran en esta reflexión como un saber del entendimiento que conforma la "esfera de la finitud" y se sitúa directamente frente al "pensamiento". Mediante esta posición metodológica, la esfera de las necesidades puede problematizarse de manera apropiada: la definición fenoménica de la esfera de las necesidades como una "infinita cantidad de singularidades" o sus "principios simples" representan las piedras temáticas angulares de la economía como ciencia particular. Sus categorías forman una herramienta adecuada para el análisis de los fenómenos en cuestión. Esta es una posición coherente en sí misma y al mismo tiempo reflejada y legitimada por la filosofía como ciencia de la razón: la filosofía "justifica” el punto de vista del entendimiento, o su ciencia de la economía "con sus puntos de vista". En el contexto de estas consideraciones, los "principios simples de la cosa" son los principios que la economía puede concebir y expresar a su manera. Es decir, los múltiples fenómenos de la economía aparecen para la economía inmediatamente como necesidades, propósitos de los sujetos en su "infinita cantidad de singularidades", a partir de los cuales la economía, desde el punto de vista del entendimiento, puede “descubrir" el “principio simple de la cosa”. Desde el principio simple surge que la economía también puede reconocer una especie de "relación y el movimiento de la masa" de los fenómenos de la economía moderna. Esta primera estructuración tiene lugar desde la perspectiva de una ciencia en proceso de análisis que puede reconocer y mostrar la esfera de las necesidades, pero no su sistema.

Por tanto, la perspectiva de la razón refleja inicialmente la economía como una ciencia particular, pero también juega un papel científicamente constitutivo: la perspectiva filosófica complejarelacional-sistemática abre la posibilidad de obtener diferentes y nuevos tipos de conocimiento sobre la economía moderna. Los términos particularidad y universalidad utilizados por Hegel como figuras lógico-filosóficas del pensamiento para problematizar las necesidades y su satisfacción 
sugiere que el sujeto no es el número infinito de singularidades en una esfera fenoménica de necesidades, como en el caso de la economía, sino más bien sus características filosóficamente tematizables. Esto no solo incluye la particularidad como figura lógica del pensamiento, sino también el derecho socio-filosóficamente entendido de cada particularidad en cuanto sujeto libre de la sociedad moderna. Esto también se aplica a la satisfacción, que aquí no significa una materialidad concreta, sino la estructura social de satisfacción o el derecho socialmente asegurado a la satisfacción de la particularidad subjetiva. Estos derechos en tanto formas normativas universales sirven como autoafirmación de todos como sujetos libres de la economía y la sociedad modernas. En este contexto, el principio de universalidad puede discutirse no solo como el derecho a la satisfacción individual, sino también como una de las estructuras sociales básicas en tanto mecanismos socioeconómicos de satisfacción.

Este tipo de terminología, como teoría sistemática de lo práctico con fundamento lógico, permite en general mirar entre bastidores la economía moderna y explicar la "verdadera" forma de satisfacer las necesidades. Esta posición filosófica ofrece la posibilidad de analizar la relación de la persona concreta con sus propias necesidades subjetivas infinitamente variadas (como una "infinita cantidad de singularidades" del nivel fenoménico de cada vida) en relación a otras personas que tienen también sus propias necesidades subjetivas infinitamente variadas y se esfuerzan por satisfacerlas. Los dos principios de la sociedad burguesa se pueden explicar precisamente por la conceptualidad de la particularidad de estas necesidades subjetivas, infinitas y diferenciadas, y por la universalidad de la relación mutua en la satisfacción de necesidades a través de estructuras y mecanismos sociales (instituciones en un sentido más amplio) como características fundamentales de las prácticas. En la perspectiva económico-filosófica de la razón constitutivo-sintética con su conceptualidad, la particularidad explica la "esencia" de las necesidades infinitas subjetivas de las personas concretas individuales, así como también muestra lo "esencial" de las relaciones de la universalidad como sociabilidad primaria de la satisfacción en general, que ahora resulta ser la forma básica de la práctica. Son precisamente las relaciones entre estos aspectos las que representan la "verdadera" estructura básica de las necesidades y su satisfacción, tanto en el mundo de la vida individual como en los mecanismos, macroestructuras e instituciones económico-sociales de la sociedad moderna (riqueza, miseria y lujo) que también resultan ser características estructurales esenciales de la economía moderna.

Hegel llega aquí a la siguiente conclusión: la economía trabaja con categorías que reducen fenómenos como la "infinita cantidad de individualidades" a los "principios simples de la cosa" en un hilo de pensamiento donde la economía procede analíticamente. Este punto de vista es el de la ciencia económica especializada producto de los tiempos modernos, pero que en última instancia puede también ser referido de manera apropiada, es decir reflejado y justificado desde el punto de vista de la filosofía (razón). Los principios simples de los fenómenos, la "infinita cantidad de individualidades" que el entendimiento "descubre", sólo explican una dimensión de la economía moderna. La posición filosófica de la razón por otro lado, es capaz de comprender la esencia de la economía moderna en términos de la estructura general de la sociedad moderna. Esto significa que i) la posición filosófica en su perspectiva general puede reflejar de manera convincente y razonable los principios simples del diverso mundo fenoménico de la economía o su ciencia; y, ii) que las estructuras y movimientos profundamente arraigados de la economía 
moderna y la sociedad según su naturaleza "verdadera" sólo puede ser examinadas, explicadas, justificadas o criticadas desde esta perspectiva.

Esta forma de interpretar la economía moderna también aporta nuevos tipos de conocimiento en comparación con la economía que procede analíticamente -precisamente porque es constitutiva. La interpretación hegeliana de los fenómenos o de los principios simples de la cosa en la economía o su conexión sistemática con los dos principios de la sociedad burguesa apunta en última instancia a que una explicación adecuada de los fenómenos y de principios de la economía moderna, sea desde el punto de vista del entendimiento y su configuración científica (la economía), o desde la razón y su cientificidad, necesita en última instancia de la filosofía económica. La composición de la exposición central del §189 expresa también esta consideración fundamental.

La teoría hegeliana de la economía moderna trae consigo sentidos provechosos en comparación con la economía. La filosofía económica puede decir algo diferente y más profundo sobre temas que en muchos aspectos son análogos, pero que la economía ni siquiera puede reconocer. Es por eso que la filosofía económica debe entenderse como una teoría alternativa y relevante de la economía moderna que Hegel no desarrolló a partir de restricciones sistemáticas, sino por buenas razones conceptuales.

\subsection{Los logros y límites de la economía como ciencia particular.}

Hegel también se refiere a los límites que subyacen a la economía como ciencia y están relacionados con las particularidades de las estructuras "efectivas" de la sociedad burguesa. También le atribuye al entendimiento la importancia de ser el "poder gobernante" de los principios simples. En este sentido, Hegel llama a la economía y la sociedad modernas el "estado del entendimiento". Es por ello que el entendimiento no es solo esa posición metodológicoteórica que toma la economía como ciencia especializada, sino un nivel estructural de la realidad de la economía y la sociedad modernas. Por supuesto, otro problema es que Hegel también usó la expresión en un sentido más amplio: “en nuestra época la economía rural se maneja también de una manera reflexiva, como una fábrica, y adopta así un carácter que se opone a su naturalidad, carácter que corresponde a la segunda clase” (1968, VII, §203ad). En consecuencia, Hegel desarrolla un potencial crítico tanto teórico-interno como socialmente externo, que también permite comparaciones adicionales con otras teorías de la economía o con la respectiva realidad de la economía moderna: ien este contexto se piensa en Marx o Lukács!

¿Cómo entender este potencial socialmente crítico? La relación recíproca de las personas concretas se entiende como una forma de universalidad en la medida que las personas no entienden la relación con los demás como su propia universalidad normativa y social en tanto eticidad y la ponen en acción en sus prácticas. Esto es así porque solo pretenden satisfacer sus propias necesidades particulares. Al mismo tiempo, deben e incluso necesitan darse cuenta de que solo pueden satisfacer sus propósitos y necesidades particulares mediante la cooperación con los demás. El "egoísmo" como posición egocéntrica del primer sujeto de la sociedad burguesa, la persona concreta, se revela, así como un déficit ético y social. Es precisamente la colisión interna entre el sujeto egocéntrico de las necesidades y la relación forzada con otros en la satisfacción lo que caracteriza el presente tipo de universalidad como una sociabilidad formal. Este tipo de intersubjetividad y sociabilidad se desarrolla por necesidad y compulsión, y no por una 
comprensión y eticidad racionales. En consecuencia, las personas concretas se instrumentalizan entre sí. En este sentido, la sociedad burguesa es en sí misma un “estado del entendimiento" en el que la actitud egocéntrica es normal. La única relación formal entre las personas concretas y la instrumentalización mutua y por tanto también la alienación es en realidad normales: son niveles elementales de una intersubjetividad y sociabilidad no desarrolladas que surgieron por necesidad y coacción. En este punto se topa con los límites de la economía como ciencia especializada, que no puede explicar estas estructuras deficitarias. En este sentido, Hegel se preocupa no solo por un nuevo potencial de interpretación, sino también por un potencial socialmente crítico.

La posición de Hegel también trae consigo un resultado teórico negativo. Este consiste en el hecho de que las estructuras y movimientos fundamentales de la economía moderna no pueden tematizarse solo con medios económicos. En otras palabras: la economía ofrece una interpretación necesaria pero insuficiente de la economía moderna. Para poder superar este déficit, la economía moderna o sus fenómenos tienen que ser interpretados en el doble horizonte de la economía y la filosofía económica. Así lo expresa la observación de Hegel en el \$189, donde que reconoce el logro de la economía, a la vez que lo critica, amplía y reinterpreta desde la perspectiva de la filosofía económica. Esto significa que la interpretación económica no solo se diferencia de la filosófica, sino que también se integra en la filosofía económica.

En realidad, puede reconocer bastante, especialmente el mundo fenoménico de las necesidades, propósitos, satisfacción o sus principios simples: son de hecho su objeto. Hegel se expresa en términos muy positivos sobre el logro científico de la economía: muchas características de la economía moderna pueden ser descritas de manera precisa por la economía mediante sus propios medios (categorías), pero no todas. La economía tiene límites y déficits teóricos internos, que la filosofía económica en su perspectiva metodológica-sistemática compleja puede revelar y superar.

Sin embargo, la filosofía económica discute la economía y la sociedad modernas no solo en términos de la base teórico-metodológica de la razón, sino también en términos de eticidad. La sociedad civil está incorporada en la eticidad como la forma más elevada de integración en el espíritu objetivo. Correspondientemente, Hegel describe la realidad de la sociedad burguesa como el mundo de la aparición de la eticidad. El “mundo de los fenómenos” no es una construcción conceptual pura, debe entenderse como un término relacionado con los fenómenos concretos que Hegel constituye no solo de acuerdo con la lógica, sino sobre todo con respecto a los fenómenos que pueden, por ejemplo, "ser estudiados a gran escala en Inglaterra" (Hegel, 1986, VII, §245; Jamme y Weisser-Lohmann 1995; Weszek, 1988). Desde la perspectiva de la normatividad ética, la economía moderna es una realidad invertida en la medida que esta realidad elude la normatividad ética. La economía no puede ver la esencia de este error, aun cuando puede reconocer fenómenos intrincados como el lujo y la miseria. Desde esa doble perspectiva de la filosofía práctica, cuyos pilares son la razón y la eticidad, la sociedad burguesa puede ser analizada y retratada como una apariencia de razonabilidad, y así también como una eticidad perdida en su naturaleza invertida, contradictoria y compleja, es decir, 'verdadera'. El potencial crítico del enfoque del filósofo surge de esta doble perspectiva, que permite entender, expresar y criticar las contradicciones y el mal del mundo fenoménico de la sociedad burguesa como sus aspectos y estructuras propias. 
Podemos decir entonces que, para desarrollar una interpretación adecuada de los fenómenos y principios de la economía y la sociedad modernas, es inevitable una forma de ciencia que sea capaz de liberar a temas (sus objetos de estudio) de las limitaciones de la metodología de una ciencia especializada. Para hacer esto, sin embargo, se necesita la transgresión del "entendimiento" como posición metodológica de una ciencia especializada. Este traspaso de fronteras permite explicar la base metodológica de la razón como una posición básica teórica diferenciadora y al mismo tiempo integradora, también en este sentido complejo-sistemático, o la explicación de la normatividad en la práctica que representa la eticidad. La transición de la economía política a la filosofía económica, el logro soberano de Hegel, tiene lugar sobre esta base conceptual. Ahora bien, la racionalidad práctica, que juega un papel decisivo en la iluminación de las estructuras de la filosofía práctica de Hegel, debe aplicarse en el área de tensión entre razón y eticidad. Hegel también incluyó necesidades, deseos, inclinaciones, etc., así como sentimientos, en la razón como un tipo específico de racionalidad. El espíritu, como sustancia ética y como subjetividad, transforma estos elementos naturales y formas interiormente subjetivas en las que se reconoce la actividad de la razón (Pippin, 2008; Halbig, 2009).

No es casualidad que Hegel busque explicaciones más profundas del mal infinito en el ámbito de las necesidades. Pero sin perseguir las ilusiones de poder negar las estructuras y características en confrontación (los “entrelazamientos”) de la economía y la sociedad modernas. El potencial crítico de su concepción de la economía y la sociedad modernas incluye la búsqueda de medios que no apunten a resolver las confrontaciones, sino que puedan mitigarse y mantenerse dentro de límites, a fin de evitar la desintegración no solo de la sociedad y la economía, sino también de los micro-mundos como el entorno de vida de los individuos. Esto se hace con la intención programática de estabilizar sus vidas, y además mejorar su calidad. Este programa no presupone actividades revolucionarias, pero presupone reformas sensatas. Uno de sus elementos sigue siendo relevante hasta el día de hoy: el mejoramiento cualitativo en el estilo de vida.

En última instancia, Hegel basó el aumento cualitativo del estilo de vida en el principio de la libertad infinitamente subjetiva de todos en la era moderna y lo vinculó a su concepción de la educación. Llevó a cabo esta concepción en la Filosofía del derecho de 1820 en relación con su teoría de la economía moderna. Al hacerlo, también problematizó una dimensión completamente nueva de la modernidad en la filosofía alemana, en abierta conexión teórica con las ideas de Lutero. Sin embargo, la vinculación de la discusión educativa alemana con la economía británica es su logro original. Esto muestra que no solo la construcción de la conexión entre razón y eticidad, sino también la inclusión de la educación como un asunto práctico-filosófico expresa una característica más e incluso una nueva dimensión de su filosofía económica autónoma. Para mostrar el logro filosófico del vínculo entre la economía y la educación, esta debe reconstruirse como un término económico-filosófico en el sentido hegeliano.

\section{La educación como concepto económico-filosófico.}

Es obvio que la expresión “educación” aparece más de diez veces en el capítulo sobre la sociedad burguesa. ¿Cómo se explica esta importancia? En primer lugar, el punto es que Hegel fue el único filósofo alemán que incluyó la dimensión económica de la sociedad moderna en la discusión 
formativa en el momento en que la economía británica en Alemania había permanecido en los márgenes del interés filosófico (Rózsa, 2007). Se esforzó por mostrar el topos y la función de la educación en el contexto de las estructuras sociales, económicas y políticas modernas, y las correspondientes formas de conocimiento, formas de vida y comportamiento. La educación se despliega como el aspecto de la filosofía práctica de Hegel, en cuyo trasfondo se puede reconocer su la teoría de la modernidad, incluida su concepción de la individualidad (Rózsa, 2012, pp. 18-21).

A continuación, nos enfocaremos en los aspectos de este concepto que dejan en claro que la concepción hegeliana de la educación no puede separarse de su interpretación de la economía moderna. Al contrario: la educación es un aspecto esencial de su filosofía económica. Si se interpreta el punto de vista de Hegel por separado de su concepción económico-filosófica, sería difícil explicar cómo y por qué asigna una función concreta y económicamente eficaz a la educación en el contexto de la "reciprocidad del trabajo", como división social de trabajo, o producción y consumo, cuando interpreta la educación como un elemento constitutivo de la riqueza. Asegurar la subsistencia de todos depende, por un lado, de la riqueza general, en la que, por otro lado, todos participan a través de su propia educación, habilidad y trabajo (Hegel, 1986, VII, §199).

Henning Ottmann afirmó en su ensayo Arbeit und Praxis bei Hegel que el siglo XIX, que se caracterizó por el contraste entre trabajo y educación, no pudo reproducir la síntesis de Hegel. Es por eso que, en la época del imperio de la tecnología, la filosofía, que comprende la maldición y la bendición del trabajo, parece tener más actualidad que nunca (1977, pp. 33-34). Ottmann señaló la importancia de la educación como categoría económica para el trabajo, pero sin continuar esta síntesis según la cual la educación misma logra esta relación con el trabajo en Hegel. A continuación, se explica esta síntesis teniendo en cuenta la posición de Hegel sobre la filosofía económica, que quedaría incompleta sin su concepción de la educación.

\subsection{Trabajo y cultura.}

La manera en la que Hegel sitúa la relación entre trabajo y educación es interesante en sí misma. Hizo de esto un tema cuando discutió algunas de las características fundamentales de la sociedad y la economía modernas en los párrafos introductorios de la sociedad burguesa, y en ese punto del texto, como se señala más arriba, se trata de presentar la perspectiva económicofilosófica hegeliana. Es más que interesante que en el \$187, en el penúltimo párrafo de la parte introductoria de la sociedad burguesa, Hegel introduzca la educación y la aborde precisamente en su relación con el trabajo. ¿Qué significa esto?

A los ojos de Hegel, el trabajo también es una categoría económica, aunque aquí no toma el trabajo como una categoría prioritariamente económica, sino solo más adelante cuando explica las categorías económicas. Lo hace en el apartado sobre “El modo del trabajo" en el capítulo sobre "El sistema de las necesidades", del mismo modo en que ya había tematizado el trabajo en relación con las necesidades y su forma socioeconómica, con el "consumo" y otras categorías económicas como “medios”, “elaboración”, “producción”, “producir”, “división del trabajo” o “maquinaria” (Hegel, 1986, VII, §196-198). Se debe llamar la atención sobre el hecho de que entre 
estos dos parágrafos Hegel analiza la educación teórica y práctica. Esta clasificación habla en sí misma también a favor de que las categorías económicas, que están tematizadas en los §§196 y 198, no pueden desligarse de la perspectiva económico-filosófica y que la educación expresa claramente en el §197. En otras palabras, las categorías económicas también se entienden en el punto central del texto desde una perspectiva económico-filosófica.

Por otro lado, en el §187, donde el foco está puesto en la relación entre trabajo y educación, Hegel entiende el trabajo definido desde la perspectiva de la filosofía económica. Se trata del concepto de trabajo determinado desde una perspectiva filosófica (económica). El concepto filosófico del trabajo surge precisamente en su relación con la educación, y no en relación con las necesidades, los medios o la producción, como cabría esperar desde una perspectiva económica. La frase clave sobre la determinación filosófica del trabajo dice así: “la educación es por lo tanto en su determinación absoluta la liberación y el trabajo de liberación superior, el punto de tránsito absoluto a la infinita sustancialidad subjetiva de la eticidad” (Hegel, 1986, VII, §187).

Esta frase clave sobre la relación entre trabajo y educación muestra cómo Hegel reinterpreta el trabajo como un término económico-filosófico. La educación es precisamente el concepto con el que implementa esta diferencia, y así también la diferenciación del contenido de la obra, que ha de ser ejemplar por las características ulteriores tanto de las categorías económicas como de los conceptos económico-filosóficos. Tampoco se puede pasar por alto que esta reinterpretación se da en torno a la eticidad, que expresa el arraigo socio-filosófico y la función socio-cultural de la educación.

¿Cuál es el significado económico y socio-filosófico de la educación? La educación para la libertad en el pensamiento de Hegel puede ayudar a explicar esto. En los párrafos introductorios sobre la sociedad burguesa donde analiza los principios y estructuras básicas de la economía y la sociedad modernas, se refiere al principio histórico más elevado, al "principio de la personalidad infinita y autónoma del individuo, de la libertad subjetiva” (Hegel, 1986, VII, \$185). Esta libertad infinitamente subjetiva del individuo en la era moderna representa el último fundamento para el desarrollo de la "existencia particular" de todos y conlleva la "diversidad" como pluralización de valores y figuras mentales o estilos de vida y comportamientos. Esta idea también es decisiva para la teoría de la individualidad moderna de Hegel (1986, VII, \$186). La libertad subjetiva moderna no es dada, sino que se entrega: la libertad subjetiva de cada uno debe ser formada por los respectivos individuos particulares, es decir que debe ser reconocida, apropiada y practicada. Debe formarse la subjetividad en su particularidad; este es el imperativo de Hegel (Hegel, 1986, VII, §187).

La educación es así la forma en la que el espíritu se encuentra in concreto en el proceso de humanización y socialización o en el proceso de individuación. Los individuos con sus particularidades son personas privadas que relacionan sus conocimientos, voluntades y actividades directamente con sus vida individual-particular, pero al mismo tiempo también con otros individuos (generalización como socialización normativa y práctica), de los que además se diferencian (particularización reflejada, mediada como individuación). Esta intensa relación entre individuos es, por un lado, una diferenciación y un desarrollo de las particularidades del individuo y, por otro, una integración en las relaciones sociales. En este proceso, el individuo 
inmediatamente particular se convierte en un individuo particular autodeterminado y seguro de sí mismo y, al mismo tiempo, en un miembro de la comunidad y de la sociedad en general. La conversión de la "vida particular" inmediata de cada individuo en una "existencia objetiva" tiene lugar precisamente a través de la educación, que cambia la vida del individuo en dos sentidos: uno privado y otro social (Hegel, 1986, VII, §187). En este sentido, la educación es tanto liberación en sí misma como un trabajo de liberación superior que consiste en un trabajo duro en cuanto “trabajo de educación” (Hegel, 1986, VII, §187). Los individuos se liberan a sí mismos a través de este trabajo duro de educación y se convierten en sujetos infinitamente libres que se dan su particularidad, "efectividad" y "objetividad" propias y educadas, y en este último sentido contingentes, como fundamentos estables de su propio entorno vital-ético y social. La fórmula "educación para la libertad" revela el significado de la educación para el modo de vida de todos y, al mismo tiempo, la función sociocultural de la educación para la integración de la vida particular de cada individuo en las estructuras sociales e institucionales de la sociedad moderna.

En este contexto, se hace evidente la atención que presta Hegel a la viabilidad práctica de la educación para la libertad en la vida de todos. Y, en consecuencia, también ve la educación en este contexto como una forma de conocimiento y comportamiento, así como una forma de realidad. La conexión de estos aspectos de la educación es decisiva para la realización práctica: este es el aspecto activo en lo humano que no es el trabajo, sino el comportamiento: "liberación es en el sujeto el duro trabajo contra la mera subjetividad de la conducta, contra la inmediatez del deseo, así como contra la vanidad subjetiva del sentimiento y la arbitrariedad del gusto" (Hegel, 1986, VII, §187).

Esta constelación conceptual de trabajo, educación y comportamiento le permite a Hegel mostrar y recomendar un modo de vida individual y al mismo tiempo socioculturalmente razonable y ético y, por lo tanto, formado para los sujetos libres de la sociedad moderna. La educación en este sentido complejo, como aquí explica Hegel en relación con el trabajo y el comportamiento, es la base de los procesos de humanización y socialización mediante los cuales cada individuo signa la naturaleza y el mundo social que inicialmente le parecen extraños pero que ahora puede producir como un mundo propio. En este complejo de humanización, socialización e individualización que se basa en la educación y se materializa directamente en el comportamiento, el trabajo no conforma una categoría meramente económica, sino un concepto socio-filosófico. (Sobre la originalidad del concepto de trabajo en Hegel, diferente a los esgrimidos por Smith y Marx: Schmidt am Busch, 2002).

\subsection{La educación como elemento estructural de la economía moderna y del mundo burgués.}

En el apartado “El modo de la necesidad y la satisfacción”, se afirma que la “multiplicación”, la "división" y el "perfeccionamiento" de las necesidades y los medios deben entenderse como factores económicos y como elementos de la educación. Sin embargo, es sobre todo un momento en el que se destaca el papel de la educación en la economía moderna. En ese contexto Hegel aborda la formación de la que emerge con claridad la transformación del trabajo como categoría de la economía en un concepto filosófico. El trabajo es un "transformación" 
del "material inmediatamente proporcionado por la naturaleza", pero la formación muestra el carácter humano de la producción. Esta perspectiva muestra el hecho de que la formación como carácter socioculturalmente formativo de la producción y la acción en general puede y debe afirmarse también en las actividades económicas. Para Hegel, ni el trabajo ni la producción representan la forma básica de las prácticas sociales, porque esa dimensión estriba en la acción, que Hegel determina inicialmente en relación con el hecho: “la cualidad moral subjetiva se refiere a la diferencia más elevada acerca de la medida en que un acontecimiento o un hecho constituyen una acción, y concierne a la naturaleza subjetiva misma” (Hegel, 1986, VII, §96). La acción así entendida se basa en la autodeterminación como máxima "cualidad moral" (Quante, 1993). Esto no es algo autoevidente para la economía y, del mismo modo, tampoco lo es la idea de que las personas deban entenderse como agentes y no solo como trabajadores desde una perspectiva económico-filosófica, lo que se produce justamente en la formación como medio de la educación.

La siguiente consideración de Hegel se relaciona con la educación teórica y práctica, que se interpretan en el \$197 como elementos estructurales propios de la economía moderna. A la formación teórica le pertenece una serie de disposiciones, porque "no consiste sólo en una multiplicidad de representaciones y conocimientos, sino también en la movilidad y rapidez del representar y del tránsito de una representación a otra, en la comprensión de relaciones complejas y universales, etc.” (Hegel, 1986, VII, §197). En la educación práctica se enfatiza la importancia del empleo, que no es lo mismo que el trabajo. El trabajo se relaciona con el material suministrado inmediatamente por la naturaleza. El empleo, por otro lado, es más que una relación con la naturaleza o el consumo abstracto: debe interpretarse en el área de tensión entre la necesidad y el hábito, donde se muestra su internalización (Hegel, 1986, VII, §197). La importancia de la cultura práctica "consiste precisamente en el hábito y la necesidad de estar ocupado" (Hegel, 1986, VII, §197ad). El hábito de la actividad objetiva tiene un valor prioritario para las actividades económicas, incluida la producción. Sin esta objetividad, que se activa como hábito en el sujeto, la habilidad subjetiva no sería suficiente para las expectativas de producción exitosa y efectiva. Es precisamente en este sentido económico y económico-filosófico que la educación práctica adquiere un estatus especial en la interpretación de Hegel de la economía y la sociedad modernas. La educación es un factor económicamente decisivo también en el sentido de que juega un papel significativo en la especialización, en la división del trabajo y en las nuevas formas de uso de las máquinas (Hegel, 1986, VII, §198).

La conexión entre estos aspectos de la educación teórica y práctica tiene que entenderse en Hegel como una especie de adecuación de los elementos del pensamiento, la acción y el comportamiento que deben utilizarse como un criterio normativo complejo para todas las actividades (económicas) en el ámbito sociocultural de modernidad. En esta compleja síntesis de trabajo y educación, Hegel vio una de las características centrales de la modernidad y su economía. Es por esa razón que la adecuación de la educación teórica y práctica es por un lado un requisito normativo-práctico que debe ser interpretado en los términos de las nuevas formas de producir y consumir y, por otro lado, se trata también de la adecuación de hábitos, necesidades, habilidades y comportamientos de los individuos que pueden planificar, realizar, corregir y cumplir sus propios planes de vida a través de elementos educativos. La educación 
se convierte así en un nivel interno de las estructuras económicas y de los macro-mundos en general, como también en el modo de vida del individuo y en sus características distintivas cualitativas.

El "estilo de vida cualitativo" es un punto de referencia enfatizado por Hegel para el estilo de vida de los individuos particulares en la era moderna, que en última instancia resulta del desarrollo de una libertad infinitamente subjetiva. Hegel conecta este estándar directamente con la educación como un "modo cultivado de conducta": "la educación supone una forma general de actuar en consideración del individuo de acuerdo a máximas y formas universales, es decir de comportarse de una manera universal” (Hegel, 1974, IV, p. 483). Ser educado es un requisito ineludible tanto para la efectividad de la economía moderna como para un estilo de vida cualitativo propio del individuo moderno. El modo de comportamiento educado debe formarse en primer lugar tanto sobre las propiedades del objeto respectivo como sobre las propiedades de los demás, es decir, actuar universalmente de acuerdo a la "naturaleza de la cosa”. En este sentido, la educación es una "atenuación de la particularidad" (Hegel, 1974, IV, p. 354) como arbitrariedad inmediata, arbitrio y contingencia. Es por eso que la educación como “objetividad" y "relación cultivada" significa un comportamiento teórico y práctico mediado culturalmente hacia las cosas y las personas. La persona concreta, como sujeto de necesidades, recibe entonces las condiciones "formales" que le "permiten realizar sus ideales", es decir, poder convertirse en una persona educada que pueda decidir libremente sobre su propia forma de vida en el sentido de la racionalidad y normas éticas. Hegel conecta esta sofisticación contextualizada social y moralmente con formas subjetivas como la disposición, el espíritu, la creencia y el comportamiento, que son lugares internos subjetivos y formas de realización de los propósitos particulares del individuo y el cumplimiento de sus propios planes de vida en general. En el espíritu práctico-objetivo no es la creencia la que tiene un valor prioritario como en la religión, ni el espíritu como en el arte, sino que, para Hegel, es el carácter el que tiene ese valor en el marco de la eticidad. El carácter como componente fundamental del habitus sociocultural, cuya ubicación subjetiva Hegel define en la "interioridad" como el "mundo interior" y la clasifica en un complejo de la conducta social "razonable" propia de los individuos modernos, es entendida por Siep como "una forma común de pensar, sentir y comportarse permanente y específica. El origen platónico de la virtud es tan tangible como el aristotélico: “el carácter es el habitus permanente de las personas concretas 'socializadas' en un grupo con determinadas funciones sociales. También es una 'forma de pensar o un nivel de reflexión”' (Siep, 1992, p. 272). Siep considera esto como uno de los principales problemas de la Filosofía del derecho de Hegel:

...el análisis sobre cómo las convicciones de los sujetos, que en última instancia sólo confían en su propia reflexión, y los grupos conformados por diferentes intereses y competencias pueden unirse para formar una voluntad común que se dirija permanentemente hacia el bien común, es el principal problema de la Filosofía del derecho de Hegel [...] ahí la característica principal de su solución está en la conexión de 'caracteres', es decir de las formas de pensar y vivir, con los roles sociales, los modos de las actividades y las 'habilidades'. Con esto pretendió unir el principio -no la fundamentación o la forma organizativa- de la vieja sociedad europea de clases con los principios de la sociedad burguesa emergente (que exigía 
igualdad ante la ley y la libre elección de ocupación) y el Estado constitucional que exige (que exigía la protección de los derechos fundamentales y la separación de poderes) (Siep, 1992, p. 270).

La educación, entendida de esta manera, es un componente prioritario de la libertad subjetiva de cada uno, incluido el mundo propio compartido con los demás, que debe estar conectado con la racionalidad y la normatividad ética. Hegel demostró este significado normativo de la educación en el sujeto ideal-típico de la economía y la sociedad modernas, es decir en el ciudadano o el ciudadano justo.

\section{El mundo burgués en la intersección de la alienación y el modo de vida.}

La persona concreta con sus necesidades infinitamente variadas como sujeto primario indeterminado de la sociedad burguesa se determina en un proceso educativo de múltiples capas para convertirse en burgués como segundo sujeto de la sociedad burguesa. En este proceso, su particularidad como actitud egocéntrica se vincula con su universalidad formal en tanto intersubjetividad (aún) no desarrollada de una manera diferente: es decir el burgués que, a diferencia de la persona concreta, tiene una autodeterminación (auto)consciente a su disposición que le permite adquirir y practicar una educación contextualizada y mediada precisamente a través de la sociedad. La autodeterminación del burgués a través de la educación como institución sociocultural universal-formal también da cuenta del principio moderno de libertad subjetiva en el mundo cotidiano común. Este proceso tiene lugar en el campo de tensión entre la vida privada subjetivamente particular y la vida sustancial-ética. Esto además se traduce en que no es la persona concreta dependiente de la necesidad, ni siquiera la persona abstracta de derecho, sino sólo el burgués educado con una autoconciencia desarrollada el que puede llevar la libertad subjetiva como uno de los valores más altos de la modernidad en la propia forma de vida racional, éticamente orientada y cultivada. En este proyecto, la "educación para la libertad" y la "satisfacción del yo" se combinan como aspectos sociales e individuales de la vida moderna y proporcionan una base para la constante diferenciación de las identidades sociales y personales, ahora frágiles debido a la constante diferenciación de las relaciones del sujeto (Rózsa, 2014b) y a la actitud "fluctuante" de los individuos modernos.

Podemos inspirarnos en las ideas de Hegel para indagar en los fundamentos éticos, normativos y racionales de las posibilidades prácticas del estilo de vida cualitativo como un problema de gran actualidad incluso si en muchos aspectos de ha perdido el tipo sociocultural ideal de Hegel, el burgués educado y justo de la modernidad clásica con sus particularidades características. Sin embargo, vale la pena investigar qué sigue siendo relevante para nuestra autoimagen a partir de este conjunto de ideas en ese momento y el nuestro, cuando las identidades altamente diferenciadas de los individuos de la modernidad tardía (o modernidad contemporánea) así como sus cuestiones de autodeterminación se han vuelto aún más complejas e incluso incomparablemente más complicadas.

Muchos aspectos de su teoría sobre las estructuras y mecanismos de la economía y la sociedad modernas o sobre el estilo de vida de los individuos modernos que está estrechamente relacionado con ella, a pesar de todas las apariencias, no han perdido nada de su actualidad. La cuestión de cuánta alienación se puede soportar, que también es relevante en este contexto, 
sigue siendo de actualidad, y tan abierta como lo fue con Hegel. (Quante, 2016). Al igual que con Hegel, la alienación es un rasgo estructural de la modernidad actual que penetra profundamente en los planes de vida, las autointerpretaciones y las decisiones de los individuos. La tesis de Hegel sigue siendo válida: la diversidad como pluralización fenoménica de las formas de vida y de los planes de vida está estrechamente relacionada con el principio de libertad infinitamente subjetiva. Estas características de la existencia moderna abren un campo infinitamente amplio, también frente a la alienación o sus fenómenos siempre renovados. La alienación aparece como un aspecto indisociable de las estructuras sociales y económicas modernas, así como forma y el estilo de vida de las personas. La cuestión de dónde se van a determinar los límites de su sostenibilidad es un desafío recurrente, que se puede ilustrar con el mito de Sísifo de Camus como un ciclo infinito, pero que difícilmente se pueda responder con seguridad.

Las afirmaciones que suenan optimistas sobre el estilo de vida cualitativo y su pluralización cruzan constantemente las inquietantes preguntas recurrentes sobre los fenómenos de alienación que se multiplican inexorablemente. No es casualidad que Hegel solo mencione marginalmente la reconciliación como principio estructurador de su filosofía práctica en la sociedad burguesa, el punto central de su filosofía económica. Las “colisiones” y los "entrelazamientos” y no su reconciliación dominan la vida moderna, del mismo modo que la "existencia particular" lo hace en el mundo de la vida individual. La profunda intuición de Hegel no ha perdido su relevancia hasta el día de hoy. La fragilidad de nuestro modo de vida en la modernidad contemporánea no se ha vuelto menor ni más débil: los medios de educación y los patrones de comportamiento, que fue donde buscó una solución, nos protegen hoy incomparablemente menos que antes (Rózsa, 2017).

\section{Referencias}

Althusser, L. (1968). Für Marx. Suhrkamp.

Brandom, R. (2002). Selbstbewusstsein und Selbst-Konstitution. Die Struktur von Wünschen und Anerkennung. In Chr. Halbig, M. Quante y L. Siep (Hrsg.), Hegels Erbe (pp. 46-77). Suhrkamp.

Brandom, R. (2001). Begründen und Begreifen. Eine Einführung in den Inferentialismus. Suhrkamp.

Habermas, J. (1986). Der philosophische Diskurs der Moderne. Zwölf Vorlesungen. Suhrkamp.

Hackenesch, Chr. (2000). Kommentar zu Die Wissenschaft der Logik (§§ 19-244). In H. Drüe (Hrsg.), Hegels Enzyklopädie der philosophischen Wissenschaften (1830). Ein Kommentar zum Systemgrundriß. (pp. 87138). Suhrkamp.

Halbig, Chrph. (2009). Das Recht des subjektiven Willens (\$132). Überlegungen zu Hegels Theorie praktischer Rationalität. Hegel-Studien 44, 95-105.

Hegel, G. W. F. (1986). Werke in zwanzig Bänden. Suhrkamp.

Horstmann, R.-P. (2014). Hegels Theorie der bürgerlichen Gesellschaft (§§ 158-256). In L. Siep (Hrsg.), G.W.F. Hegel: Grundlinien der Philosophie des Rechts (pp. 193-216). Klassiker Auslegen.

Hösle, V. (2005). Was kann man von Hegels objektiv-idealistischer Theorie des Begriffs noch lernen, das über Sellar's, McDowells und Brandoms Anknüpfungen hinausgeht? Allgemeine Zeitschrift für Philosophie, 30(2), 139-158.

Jamme, Chr., \& Weisser-Lohmann, E. (Hrsg.). (1995). Politik und Geschichte. Zu den Intentionen von G.W.F. Hegels Reformbill-Schrift (Hegel-Studien 35). Bouvier Verlag.

Lukács, G. (1948). Der junge Hegel und die Probleme der kapitalistischen Gesellschaft. Europa.

Moyar, D. (2002). Die Verwirklichung meiner Autorität. Hegels komplementäre Modelle von Individuen und Institutionen. In Chr. Halbig, M. Quante y L. Siep (Hrsg.), Hegels Erbe (pp. 209-253). Suhrkamp. 
Ottmann, H. (1977/78). Arbeit und Praxis bei Hegel. Hegel-Jahrbuch.

Pinkard, T. (2001). Tugend. Moral und Sittlichkeit. Von Maximen zu Praktiken. Deutsche Zeitschrift für Philosophie, 49(1), 65-88. https://doi.org/10.1524/dzph.2001.49.1.65

Pippin, R. (2008). Hegel's Practical Philosophy. Rational Agency as Ethical Life. Cambridge University Press.

Pippin, R. (2002). Hegels praktischer Realismus. Rationales Handeln als Sittlichkeit. In Chr. Halbig, M. Quante y L. Siep (Hrsg.), Hegels Erbe (pp. 295-323). Suhrkamp.

Pippin, P. (2001). Naturalität und Geistigkeit in Hegels Kompatibilismus. Deutsche Zeitschrift für Philosophie, 49(1), 45-64. https://doi.org/10.1524/dzph.2001.49.1.45

Priddat, B. (1990). Hegel als Ökonom. Duncker \& Humblot.

Quante, M. (2016). Handlung, System der Bedürfnisse und Marktkritik bei Hegel und Marx. In H-C. Schmidt am Busch (Hrsg.), Die Philosophie des Marktes (pp. 153-175). Meiner.

Quante, M. (2014). Die Logik ist das Geld des Geistes. Zur Rezeption der Hegelschen Logik im Linkshegelianismus und der Kritik der politischen Ökonomie. In A. Koch (Hrsg.), Hegel - 200 Jahre Wissenschaft der Logik (pp. 413-431). Meiner.

Quante, M. (1993). Hegels Begriff der Handlung. Frommann-Holzboog.

Riedel, M. (1969). Studien zu Hegels Rechtsphilosophie. Suhrkamp.

Riedel, M. (1972ff). Rehabilitierung der praktischen Philosophie. Rombach.

Riedel, M. (1975). Materialien zu Hegels Rechtsphilosophie. Suhrkamp.

Rózsa, E. (2017). Hegels Wirtschaftsphilosophie in seiner Rechtsphilosophie von 1820. In I. De Gennero (Hrsg.), Wirtliche Ökonomie. Philosophische und dichterische Quellen (pp. 283-317). Bautz.

Rózsa, E. (2014a). From Love to Recognition. Hegel's Conception of Intersubjectivity in a DevelopmentalHistorical Perspective. In Chr. Krijnen (Hrsg.), Recognition - German Idealism as an Ongoing Challenge (pp. 287-309). Brill Publisher.

Rózsa, E. (2014b). Personale und soziale Identitäten im Hinblick auf Selbstrelationen bei Hegel. HegelJahrbuch 2014(1), 260-270. https://doi.org/10.1515/hgjb-2014-0143

Rózsa, E. (2012). Modern Individuality in Hegel's Practical Philosophy. Brill Publisher.

Rózsa, E. (2007). Einleitung. En: K. Engelhard y M. Quante (Hrsg.), Hegels Konzeption praktischer Individualität, von der Phänomenologie des Geistes zum enzyklopädischen System (pp. 7-18). Mentis Verlag.

Rózsa, E. (2005). Versöhnung und System. Zu Grundmotiven von Hegels praktischer Philosophie. W. Fink.

Rózsa, R. (1993). Hegel gazdaságfilozófiája. Akadémiai Kiadó.

Schmidt am Busch, H.-Chrph. (2002). Hegels Begriff der Arbeit. Akademie.

Siep, L. (2010). Aktualität und Grenzen der praktischen Philosophie Hegels. W. Fink.

v Siep, L. (1992). Praktische Philosophie im Deutschen Idealismus. Suhrkamp.

Tillich, P. (1962). Die verlorene Dimension. Not und Hoffnung unserer Zeit. Furche.

Vieweg, K. (2019). Hegel. Der Philosoph der Freiheit. C.H. Beck.

Waszek, N. (1988). The Scottish Enlightenment and Hegel's Account of Civil Society. Kluwer Academic Publisher.

Weisser-Lohmann, E. (2011). Rechtsphilosophie als praktische Philosophie. Hegels Grundlinien der Philosophie des Rechts und die Grundlegung der praktischen Philosophie. W. Fink.

\section{AUTORA}

Erzsébet Rózsa. Profesora emérita de la Universidad de Debrecen, Hungría, donde ha sido investigadora, profesora asociada y titular desde 1970. Ha sido profesora invitada en la Universidad de Münster, Alemania. Doctora y Habilitación en Filosofía por la Universidad de Debrecen y Doctora en Filosofía económica por la Academia Húngara de Ciencias. 